\title{
Cómo evitar la lesión de vía biliar en colecistectomía laparoscópica: Más allá de la visión crítica de seguridad
}

\author{
César Muñoz C. ${ }^{1,2}$, Martín Inzunza ${ }^{1,3}$, Carlo Marino ${ }^{1,2,3}$ y Jorge Martínez ${ }^{3}$
}

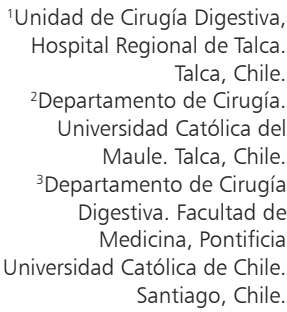

'Unidad de Cirugía Digestiva, Hospital Regional de Talca. Talca, Chile.

Departamento de Cirugía. Universidad Católica de Maule. Talca, Chile. 3Departamento de Cirugía

Digestiva. Facultad de Medicina, Pontificia Universidad Católica de Chile. Santiago, Chile.

Recibido 2020-02-07 aceptado 2020-08-31

Correspondencia a: Dr. César Muñoz C. cesarmunozcastro@gmail.
How to avoid bile duct injury in laparoscopic cholecystectomy: Beyond the critical safety view

Laparoscopic cholecystectomy (LC) is the standard of treatment for benign pathologies such as cholelithiasis and gallbladder polyps. The LC is one of the most frequent procedures in general surgery, with a $0.1 \%-0.3 \%$ associated risk of bile duct injury. Currently, scientific society programs, surgical strategies, and new technologies allow us to reduce the risk of bile duct injuries and to increase the clinical safety of this procedure. This document aims to review the strategies, technologies, and tactics to carry out a safe LC beyond the critical vision of security.

Key words: laparoscopic cholecystectomy; bile duct; bile duct injury.

\section{Resumen}

La colecistectomía laparoscópica (CL) es el estándar de tratamiento para la patología benigna como la colelitiasis y los pólipos de la vesícula biliar y es uno de los procedimientos más frecuentes de la cirugía general. Esta intervención tiene un riesgo de $0,1 \%-0,3 \%$ de causar una lesión quirúrgica de la vía biliar (LQVB). En la actualidad, existen programas de sociedades científicas, estrategias de abordaje y tecnologías que nos permiten reducir las LQVB mejorando la seguridad clínica de este procedimiento. El objetivo de este documento es realizar una revisión de las estrategias, tecnologías y maniobras para realizar una CL segura más allá de la visión crítica de seguridad.

Palabras clave: colecistectomía laparoscópica; vía biliar; lesión de vía biliar.

\section{Introducción}

La colecistectomía laparoscópica (CL) es el estándar de tratamiento de la colelitiasis y pólipos vesiculares. En sus inicios, la CL tuvo un aumento en la incidencia de lesiones quirúrgicas de la vía biliar (LQVB) por sobre la colecistectomía por laparotomía hasta una estabilización del riesgo en un $0,1 \%-0,3 \%{ }^{1,2}$.

Actualmente se han expandido las indicaciones de CL a pacientes más complejos sin mayor aumento de la morbilidad ${ }^{3}$. Junto a lo anterior, el desarrollo de programas de prevención de LQVB ha dado mayor seguridad a este procedimiento ${ }^{4,5}$.

El objetivo de este documento es revisar algunas estrategias, técnicas y herramientas para la práctica segura de la CL y se describe cómo obtener la visión crítica de seguridad y señalar las tácticas para disminuir la morbilidad en casos difíciles.

\section{Consideraciones anatómicas}

La vesícula biliar (VB) se encuentra en la fosa vesicular, concavidad hepática del segmento IVB-V de Couinaud ${ }^{6}$. Posee una cara libre cubierta por peritoneo que se continúa con la cápsula de Glisson y otra cara adherida al parénquima hepático, por donde mantiene drenaje venoso con el parénquima del lecho vesicular (Figura 1). El drenaje linfático es por una red linfática que se inicia en el ganglio cístico ${ }^{7}$.

En 1891, Jean-François Calot describió un triángulo anatómico relativamente constante conformado por el conducto cístico, el hepático común y el borde 
hepático. En este triángulo se identifica la arteria hepática derecha, la arteria cística y el ganglio cístico. Actualmente, se acepta el término triángulo hepatocístico como nomenclatura internacional ${ }^{8}$ (Figura 2).

\section{Variantes de la vesícula biliar}

$\mathrm{Su}$ posición puede variar existiendo vesículas en los segmentos VI y III, parcial o completamente rodeadas de parénquima hepático (intrahepática) ${ }^{6}$. El pedículo hepático y las ramas de la vena hepática media tienen un diámetro significativo aproximadamente a $1 \mathrm{~cm}$ de profundidad de la VB.

La VB se divide en fondo, cuerpo y cuello o infundíbulo. La bolsa de Hartmann es una dilatación de la pared vesicular en el cuello. Se le conoce también como bacinete vesicular. El bacinete puede ser redundante y cubrir el conducto cístico o el triángulo de Calot $^{6}$. En un conducto cístico corto la tracción de la VB puede hacer que el colédoco simule un conducto cístico. Una forma evolutiva es el Síndrome de Mirizzi, en el que un cálculo grande se adhiere y erosiona la pared de la VB y el conducto hepático formando una fístula colecistobiliar. El síndrome de Mirizzi se asocia a mayor dificultad de la CL.

\section{Variantes de la anatomía arterial}

La arteria cística tiene su trayecto habitual en el triángulo de Calot en un $76 \%$ de la población y en el $80 \%$ proviene de la arteria hepática derecha. La artería cística tiene 2 ramas terminales, una superficial y otra profunda, que discurren en forma paralela al cuerpo vesicular ${ }^{18}$. En el $10 \%$ de la población, la arteria cística no está en el triángulo de $\mathrm{Calot}^{19}$. Cuando la arteria hepática derecha (AHD) proviene de la arteria mesentérica superior, la arteria cística sigue siendo rama de la AHD y discurre a la derecha del bacinete próxima al surco de Rouviere?

\section{Variantes de la vía biliar}

La vía biliar extrahepática normal está formada por los conductos hepáticos derecho e izquierdo que se unen para formar el conducto hepático común (CHC) fuera de la cápsula de Glisson; 2-3 cm distal se une el conducto cístico (CC) en su cara lateral externa. El $60 \%$ de la población tiene esta disposición de la vía biliar8.

$\mathrm{El} \mathrm{CC}$ tiene tres variaciones comunes: la primera es una inserción baja, en el tercio distal del $\mathrm{CHC}$ $(9 \%)$; la segunda es la inserción medial a la izquierda del CHC (17\%); la tercera es el curso paralelo al $\mathrm{CHC}$ por trayecto de $2 \mathrm{~cm}$ o más $(25 \%)$, conocido como cístico en cañón de escopeta ${ }^{10}$.

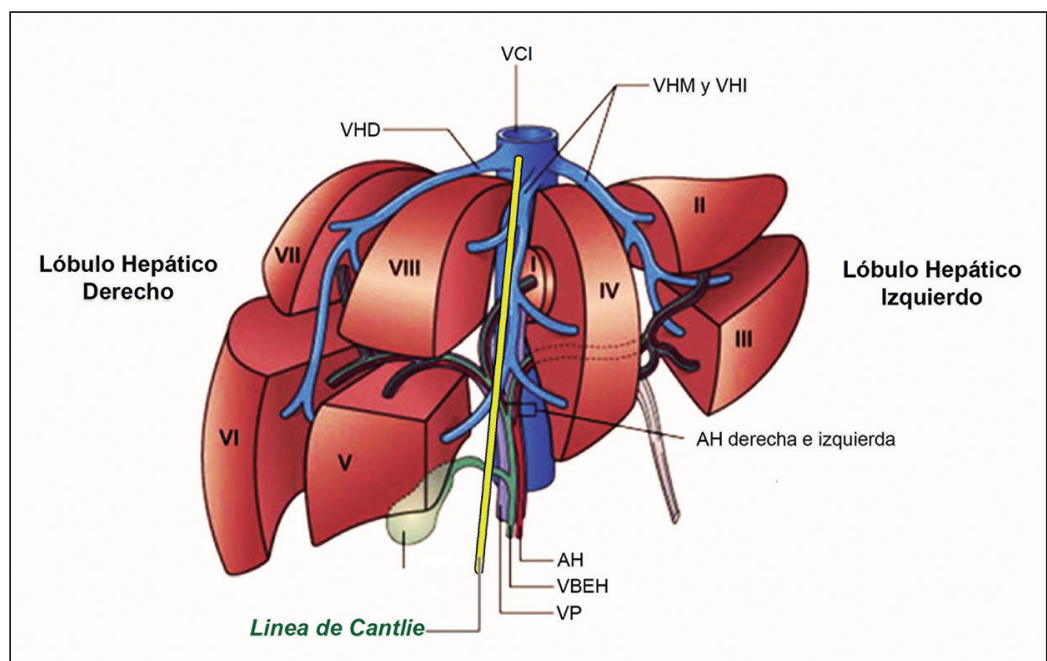

Figura 1. Esquema que muestra la anatomía hepática más frecuente y la segmentación hepática de Couinaud. VCl: Vena cava inferior, VHD: Vena suprahepática derecha, VHM: Vena suprahepática médica, VHI: Vena suprahepática izquierda, AH: Arteria hepática, VBEH: Vía biliar extrahepática, VP: Vena porta.

Otra variante es el conducto colecisto-hepático (conocido como conducto de Luschka). Este conducto puede unirse a la VB en cualquier punto de la fosa vesicular ${ }^{16}$. La incidencia exacta no está documentada y algunos cuestionan su existencia ${ }^{17}$. Un conducto biliar en la fosa vesicular es probablemente un conducto superficial y se puede ligar con seguridad. No debe confundirse con el conducto biliar derecho posterior en la cisura de Rouviere, que en una colecistitis aguda puede estar en contacto con el bacinete y ser objeto de LQVB ${ }^{11}$.

La gran variabilidad anatómica de esta región debe considerarse para evitar una confusión y sufrir una desorientación espacial ${ }^{12}$.

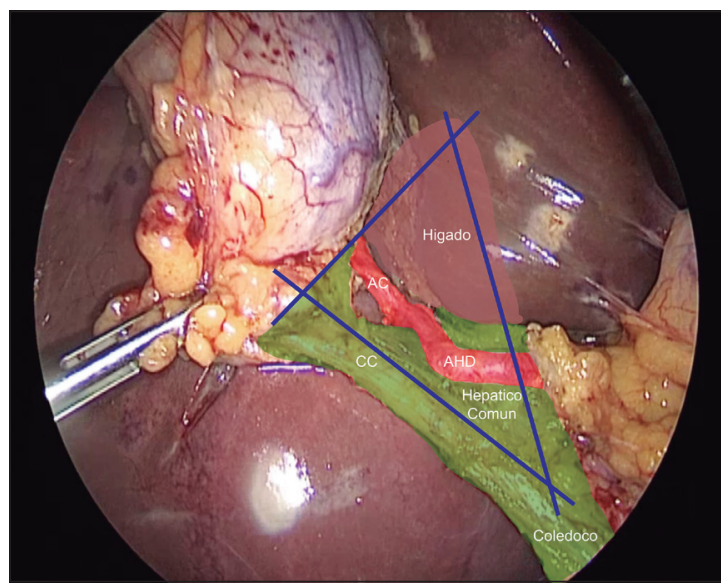

Figura 2. "Triángulo de Calot o triángulo cisticohepático en nomenclatura internacional". En esta fotografía esquemática se muestran los límites del triángulo de Calot con sus respectivas estructuras anatómicas en una $\mathrm{CL}$. 


\section{CIRUGíA AL DÍA}

\section{Técnica quirúrgica}

La CL se describe con 2 técnicas de posición de trocares, equipos y disección. A nuestro parecer, la técnica francesa tiene ventajas por sobre la americana ya que la posición de los trocares permite una mayor triangulación y la utilización de las 2 manos del cirujano. Por otra parte, la cirugía compleja hepatobiliar se realiza en posición de Lloyd-Davis y, si la CL se complica, el paciente ya se encuentra en la posición más adecuada para solucionar el problema.

Se han descrito maniobras y técnicas alternativas para dar mayor seguridad a la sección de estructuras y nos referiremos a ellas.

\section{Visión crítica de seguridad}

La visión crítica de seguridad (VCS), introducida por Strasberg et al. ${ }^{13}$ en 1995, es la estrategia más aceptada para la identificación de la arteria y el conducto cístico. La masificación de esta técnica en el intraoperatorio ha disminuido la incidencia de
LQVB en diversos estudios ${ }^{5,13-16}$. Otros estudios analizaron los mecanismos de LQVB, y demostraron que en gran parte de ellos no se realizó la $\mathrm{VCS}^{15,16}$. Para obtener la VCS se deben cumplir 3 principios ${ }^{13}$ :

- Liberación del triángulo de Calot de la grasa y el tejido fibroconectivo. La vía biliar y el conducto hepático común no deben ser expuestos en esta maniobra (Figura 3).

- El tercio inferior de la VB debe ser separada del hígado para exponer el lecho vesicular o placa cística. Honda et al. ${ }^{17}$, demostraron que esta maniobra permite obtener varios milímetros que amplían el espacio del triángulo de Calot (Figura 4).

- Dos estructuras deben ser visualizadas entrando a la VB en forma paralela: el conducto cístico y la arteria cística.

La VCS debe ser confirmada en dos planos, anterior y posterior, con la óptica en planos contrarios ${ }^{13}$ (Figura 5).

Figura 3. "Apertura del peritoneo y disección del cuello vesicular". A: En esta fotografía se muestra la apertura del peritoneo por encima de la unión cístico vesicular que permite la exposición del cuello". B: Disección del peritoneo parietal de la cara lateral de la vesícula como primera maniobra.

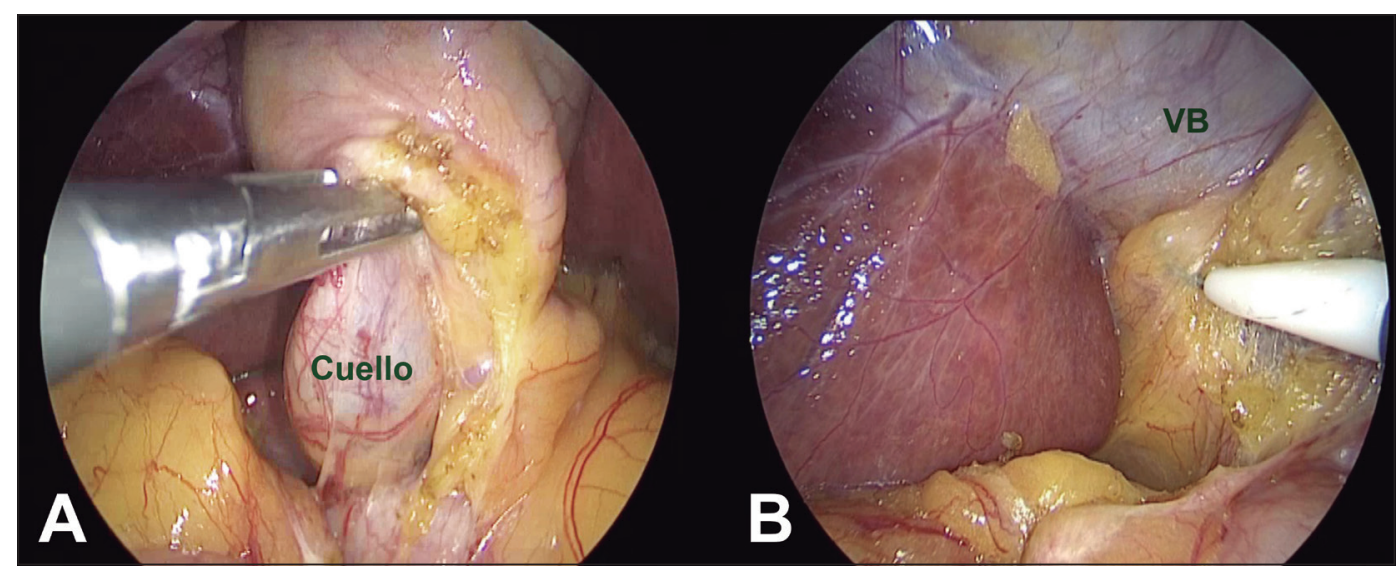

Figura 4. "Disección de la cara lateral y medial del Calot". A: Disección de la cara lateral de la vesícula biliar. Esta disección permite ganar 3-5 mm como ha demostrado Honda G, et al., en la apertura del Calot que faci litan la disección por la cara medial y con muy pocas variantes anatómicas constituyendo una región de menor riesgo. B: Sección del peritoneo por cara medial hasta el 1/3 medio de cuerpo vesicular para poder separar con mayor facilidad el cuello de lecho hepático.

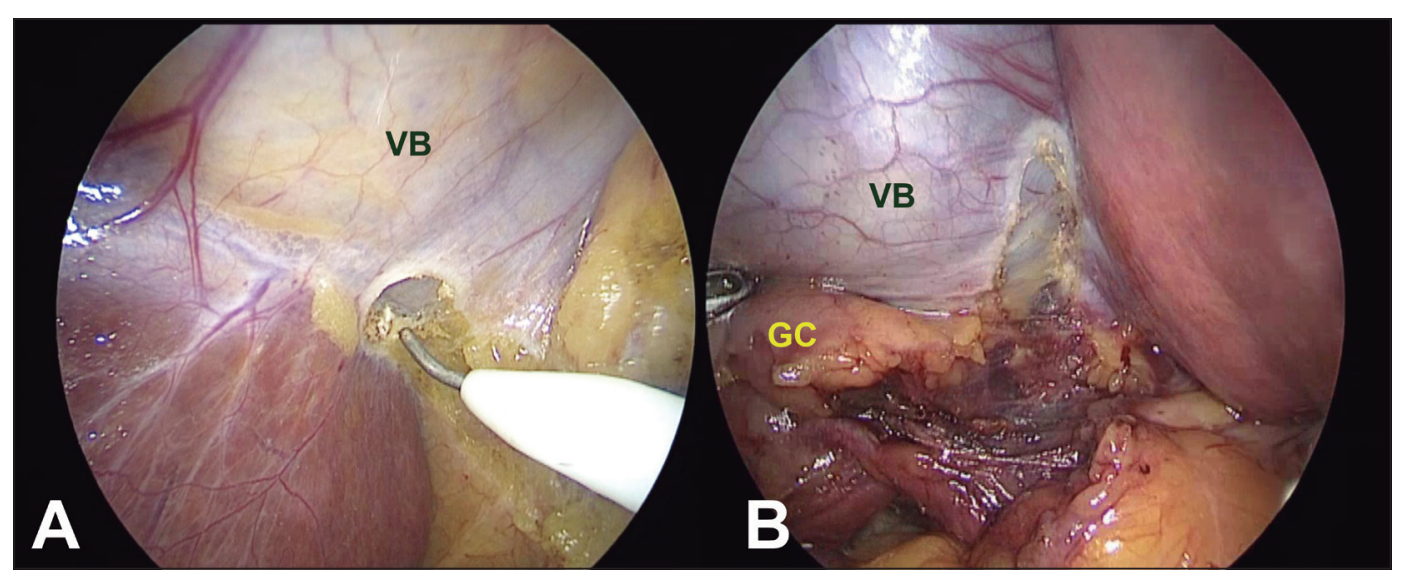




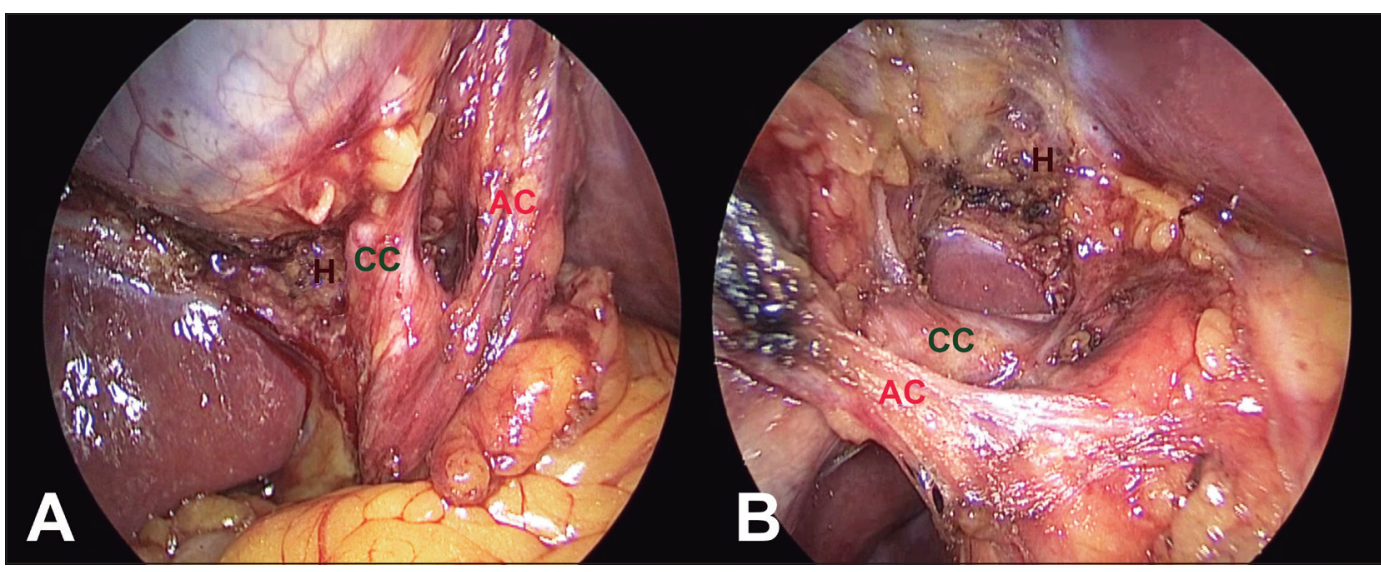

Figura 5. "Visión crítica de seguridad de Strasberg". La visión critica de seguridad se debe comprobar desde la visión directa en planos contrarios en A: derecha a izquierda y en B: izquierda a derecha. Las letras señalan $\mathrm{H}$ : Lecho hepático, AC: Arteria cística y CC: Conducto cístico.
La VCS no se puede obtener en todos los casos, principalmente por distorsión de la anatomía normal en inflamación aguda o crónica con fibrosis ${ }^{5,13,14,18}$. Si no se ha logrado la VCS debe haber una señal de alerta por una situación de mayor riesgo.

Las guías clínicas de Tokyo $^{14}$ de 2018, recomiendan 6 pasos para aumentar la seguridad durante una CL. Estas recomendaciones integran los principios anteriores para lograr finalmente la VCS (Tabla 1).

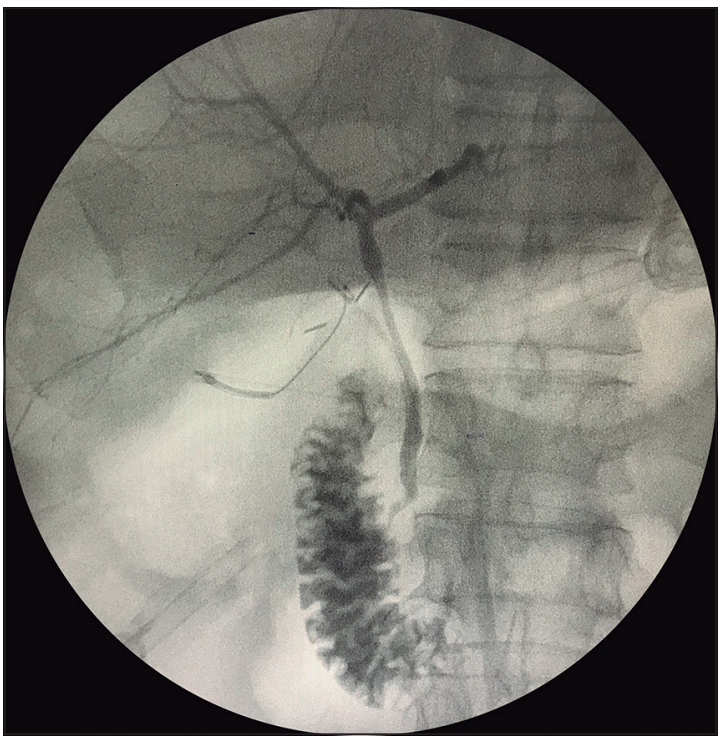

Figura 6. Colangiografía transcística estándar en una CL con una anatomía normal de la vía biliar. En este procedimiento se debe ver completamente la vía biliar intra y extrahepática sin fugas del contraste ni defectos del llene a ningún nivel finalizando con un paso fácil del contraste al duodeno.

\section{Colangiografía intraoperatoria}

La colangiografía intraoperatoria (CIO) permite el diagnóstico intraoperatorio de coledocolitiasis ${ }^{4,5,19}$ y realizar tratamiento de los cálculos incidentales ${ }^{19}$. Pero una de las indicaciones más aceptadas es el diagnóstico de cualquier fuga biliar durante la colecistectomía y la evaluación anatómica de la vía biliar ante una duda durante la disección o posterior a la sección de un conducto. En algunos centros, la utilización de la CIO es rutinaria en todas las colecistectomías laparoscópicas (Figura 6). La evidencia actual no sustenta el uso rutinario para la detección de coledocolitiasis en pacientes de bajo riesgo, sin embargo, existe evidencia a favor de su utilización en disminuir el riesgo de LQVB y detectarlas precozmente ${ }^{5,18,19}$. Hay 2 factores que

Tabla 1. Pasos seguros para CL en colecistitis aguda propuestos por las Guías de Tokyo 2018

1 Si la vesícula está distendida e interfiere con la visión del campo, se debería descomprimir mediante punción con aguja y aspiración

2 Tracción/contratracción efectiva de la vesícula para desarrollar un plano adecuado en el área del triángulo de Calot e identificar sus límites

3 Iniciar la disección empezando desde la hoja posterior del peritoneo que cubre el cuello de la vesícula, y exponiendo la superficie vesicular por sobre el surco de Rouvière

4 Mantener el plano de disección sobre la superficie vesicular durante toda la CL

5 Disecar al menos el tercio inferior del lecho vesicular para obtener la VCS

6 Generar la VCS

* En caso de sangrado persistente, favorecer la hemostasia principalmente por compresión y evitando el uso excesivo de electrocoagulación o clips 
juegan en contra de su utilización rutinaria, pero que pueden mejorar con su práctica reiterada: primero, el tiempo quirúrgico que agrega una $\mathrm{CIO}$ a la $\mathrm{CL}$ es en promedio $15 \mathrm{~min}^{20}$; y segundo, la experiencia en la interpretación de las imágenes por el cirujano, quien estará más familiarizado cuanto más frecuentemente la realice.

En el contexto de una colecistitis aguda o de una manejada médicamente (enfriada), algunos estudios han mostrado un rol protector de la CIO con respecto al desarrollo de $\mathrm{LQVB}^{4,19}$. Creemos que su utilización en este escenario clínico debe ser rutinaria, aun cuando la calidad de la evidencia sea insuficiente $8,19,20$

\section{Considerar la utilización de verde de indiocianina}

El verde de indocianina (IGC) es un colorante hidrofílico inyectable por vía endovenosa. Presenta un paso mínimo al intersticio debido a su alta afinidad con las proteínas plasmáticas logrando una gran concentración intravascular y se excreta por la bilis. Al ser expuesto a un espectro de luz cercano al infrarrojo (780-830 nm) y ser captados por ópticas especiales, las estructuras que presentan el contraste emiten fluorescencia que puede ser evaluada en directo a través de una fusión de imágenes en el equipo de laparoscopía, toracoscopía o un exoscopio.

De esta forma permite clarificar la anatomía de estructuras vasculares, biliares, pedículos de órganos y, a través de isquemia selectiva, delimitar áreas de resección según perfusión ${ }^{4,21,22}$.

$\mathrm{Su}$ uso como colangiografía fluorescente fue descrito en 2009, pudiendo ser repetida gracias a su facilidad técnica y escasa toxicidad. Se han descrito tasas de visualización de $71 \%$ a $100 \%$ del conducto cístico, $33 \%$ a $100 \%$ del conducto hepático común, 25 a $100 \%$ de la unión cístico-hepático común y entre $50 \%$ y $100 \%$ del colédoco ${ }^{4}$. En pacientes donde la $\mathrm{CIO}$ resultó frustra, se reportó tasas de éxito de un $80 \%$ para identificar la anatomía biliar extrahepática $^{19,23}$.

La costo-efectividad de la utilización de IGC en una CL en comparación con la CIO aún no están claros. Recientemente, un ensayo clínico aleatorizado, demostró que la utilización sistemática de IGC es superior a la cirugía habitual ( $\sin$ IGC) para la identificación de estructuras biliares críticas en una $\mathrm{CL}^{24}$.

En nuestro medio esta tecnología aún no se ha masificado, probablemente será parte del arsenal habitual del cirujano en un futuro cercano (Figura 7).

\section{Pausa quirúrgica y solicitar una segunda opinión}

En el intraoperatorio y antes de clipar o cortar cualquier estructura, si la anatomía no es clara y segura, se recomienda realizar una "pausa". Esta pausa o time-out tiene la función de propiciar una
Figura 7. $\mathrm{CL}$ utilizando fluorescencia con verde de indiocianina. A: Fluorescencia monocromática previa disección del calor y posterior a disección del Calot. B: Imagen de fusión de fluorescencia previa disección del Calot y posterior a disección del Calot (gentileza del Prof. Enrique Lanzarini).

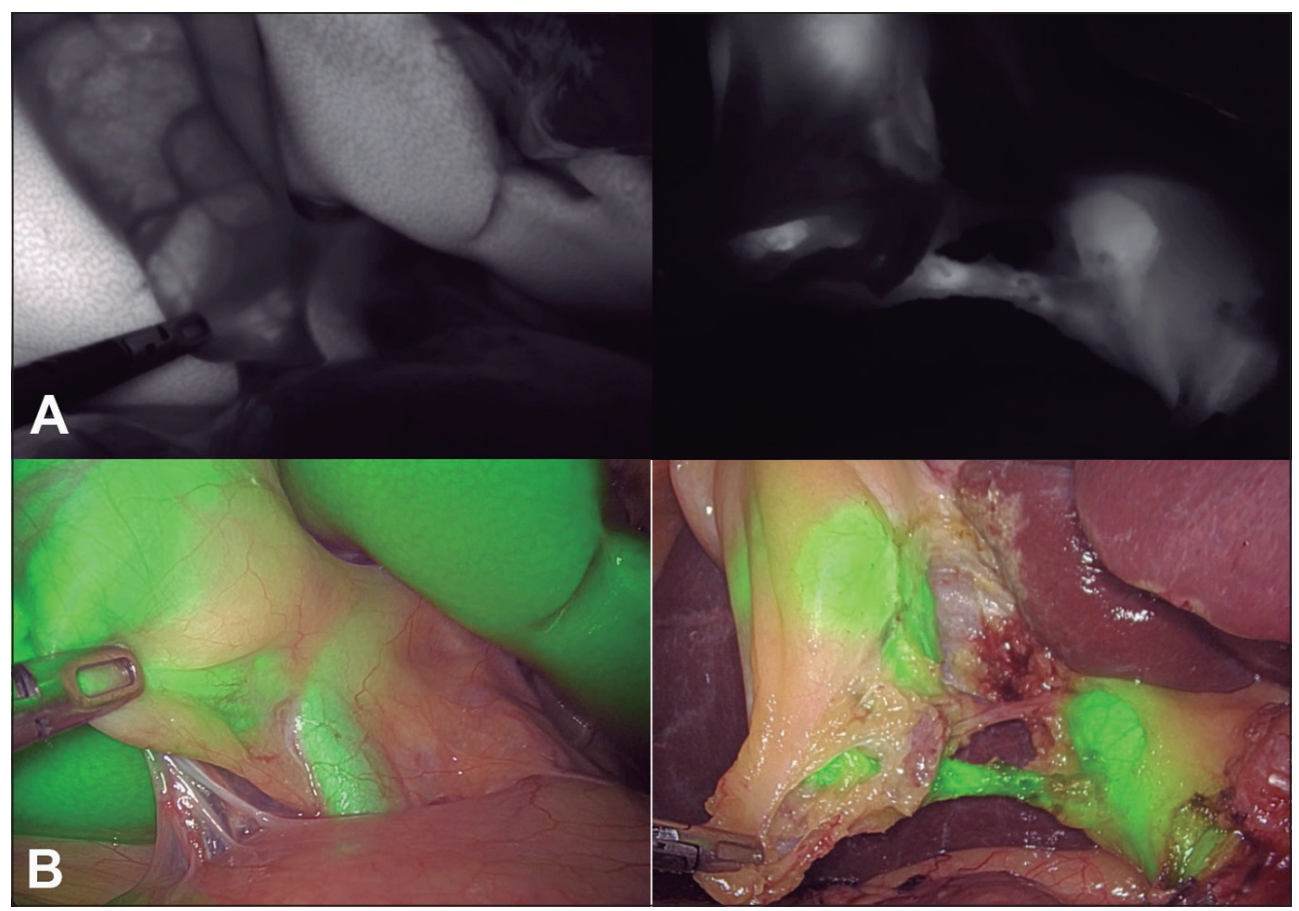


mirada con calma de la situación quirúrgica y actuar en consecuencia con ello.

Esta pausa intraoperatoria, ya es parte de las recomendaciones de la guía SAGES (Society of American Gastrointestinal Endoscopic Surgeons) para la prevención de $\mathrm{LQVB}^{25}$, y se recomienda una pausa de visión crítica en los estudios prospectivos.

Si la VCS no se ha logrado a pesar de las medidas descritas, las guías aconsejan llamar a otro cirujano para consultar su opinión o solicitar ayuda. El cirujano debe ser criterioso y solicitar ayuda antes de realizar cualquier paso irreversible.

\section{Considerar conversión a laparotomía y otras técnicas}

La conversión a laparotomía es una de las alternativas más recurridas para evitar la LQVB en situaciones difíciles al tener mejor exposición, poder palpar y sentir de manera directa las estructuras anatómicas. La conversión a laparotomía es una alternativa cómoda en escenarios difíciles, sin embargo, cada vez es menos practicada por las nuevas generaciones de cirujanos haciéndolos inexpertos en casos difíciles. Un proceso inflamatorio agudo o crónico reagudizado, con retracción y fusión inflamatoria de la vía biliar/cístico es donde la identificación manual de las estructuras es capaz de disminuir el riesgo de $\mathrm{LQVB}^{7,8,18}$. Otra circunstancia donde la conversión es adecuada es un sangrado incontrolable $\mathrm{y}$ adherencias rígidas a colon transverso o duodeno. Cirujanos con habilidades laparoscópicas avanzadas pueden resolver gran parte de estos problemas sin tener que convertir.

\section{Colecistectomía parcial o subtotal}

La colecistectomía subtotal es una alternativa en el curso de una CL difícil, y puede realizarse por vía laparoscópica o abierta. Según la guía SAGES las indicaciones para colecistectomía subtotal son las mismas que para una conversión, particularmente al no lograr una VCS. En estos casos se debe realizar una colecistectomía "en directa" (de fondo a bacinete) y si no es posible identificar las estructuras, proceder a la sección de la vesícula sobre el bacinete. Hay dos formas de realizar la colecistectomía parcial, la colecistectomía subtotal fenestrante o reconstituyente ${ }^{26,27}$. Ambas técnicas comparten la fase resectiva y consiste en la disección parcial del cuello en directa (Figura 8). En esta disección se debe tener precaución al acercarse al hilio hepático para evitar una tracción del confluente biliar. Al llegar al cuello vesicular se debe seccionar transversalmente la vesícula biliar y extraer el contenido intralumi-

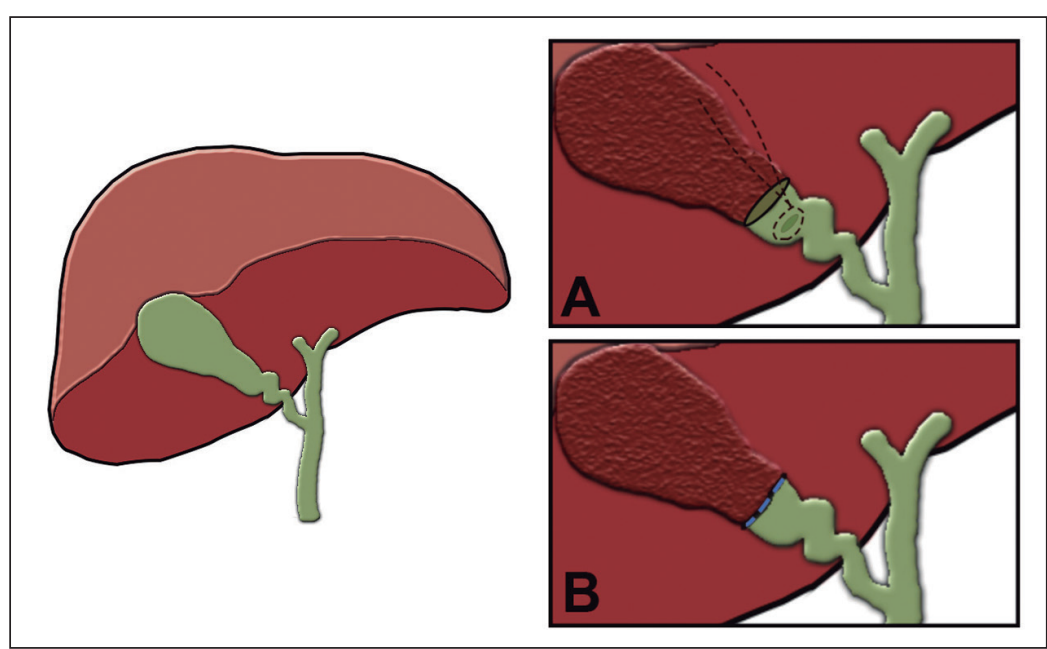

Figura 8. Esquema que muestra las 2 técnicas de colecistectomía subtotal. A: Colecistectomía subtotal "fenestrante" donde el conducto cístico se cierra por vía endoluminal. B: Colecistectomía subtotal "reconstituyente" en la cual se sutura la pared del remanente vesicular.

nal. La técnica fenestrante hace referencia a dejar la mucosa de la VB comunicada con la cavidad peritoneal, realizando un cierre del conducto cístico con una gareta por vía intraluminal. Recomendamos la utilización de un drenaje en el muñón suturado, por el riesgo de filtración biliar. Existe una variante técnica de la colecistectomía subtotal fenestrante o colecistectomía de Pribram, en esta técnica la vesícula se secciona longitudinalmente resecando su cara anterior y dejando la cara adherida al hígado evitando el riesgo de un sangrado excesivo particularmente en cirróticos con hipertensión portal ${ }^{28}$. Se recomienda la electrocoagulación de la mucosa de la pared posterior abandonada para evitar la secreción de mucina en el posoperatorio, situación que ha sido descrita en la técnica fenestrante ${ }^{28}$.

\section{Colecistostomía}

La colecistostomía es una opción en los casos donde se puede comprometer gravemente la anatomía o la vida del paciente. Se puede realizar la colecistostomía del fondo con una sonda Pezzer y dejar drenajes. Esta solución, si bien no es definitiva, permite programar la colecistectomía en un segundo tiempo con mejores condiciones técnicas y locales.

\section{Discusión}

En base a los resultados de registros prospectivos se ha establecido que la CL tiene un mayor riesgo 
de LQVB durante el periodo de aprendizaje de la técnica. Actualmente, hay estrategias institucionales y educacionales que han permitido disminuir su incidencia ${ }^{29,30}$. Paradójicamente, habiendo superado las $200 \mathrm{CL}$, vuelve a aumentar la incidencia de $\mathrm{LQVB}^{31}$.

La LQVB es una complicación conocida por todos los cirujanos, con series actuales que describen una incidencia de 1-3 casos por cada $1.000 \mathrm{CL}$ $(0,1 \%-0,3 \%)$, con mayor incidencia en cirugías de urgencia $^{32}$. La LQVB puede conducir a un deterioro en la calidad de vida del paciente, múltiples reintervenciones, conflictos médico-legales y un aumento de los costos en salud ${ }^{33-35}$. El impacto es significativo para el tratante, y su reparación tiene mejores resultados que cuando es realizada por un especialista en cirugía hepatobiliar. Nuestra recomendación es que la reparación y/o reconstrucción definitiva sea realizada por un equipo clínico familiarizado en el tratamiento de este tipo de lesiones ${ }^{36,37}$. Si no existe un especialista disponible cuando el diagnóstico de LQVB se realiza en el intraoperatorio, es preferible diferir la reparación.

La prevención de la LQVB ha conducido a la confección de guías clínicas por diferentes sociedades. Las guías de SAGES 2018 $8^{25,38}$ concentran una serie de recomendaciones con adecuado nivel de evidencia, que las hacen reproducibles a nivel global. Otras guías similares recalcan los mismos pilares fundamentales.

En este documento, hemos recordado la anatomía quirúrgica normal a una colecistectomía, y revisado diversas herramientas y estrategias quirúrgicas de utilidad para salir adelante en un momento de dificultad intraoperatoria. En la actualidad, la conversión a laparotomía puede ser un acto osado en cirujanos jóvenes con poca experiencia en esta forma de realizar una colecistectomía. En este sentido, la práctica quirúrgica y la formación de nuevos cirujanos, debe comprometer a equipos intergeneracionales donde se aporten estas experiencias.

\section{Responsabilidades éticas}

Protección de personas y animales. Los autores declaran que para esta investigación no se han realizado experimentos en seres humanos ni en animales.

Confidencialidad de los datos. Los autores declaran que en este artículo no aparecen datos de pacientes.

Conflictos de interés: no hay.

\section{Bibliografía}

1. Altieri M, Brunt M. Elimination of Bile Duct Injury in Cholecystectomy. Adv Surg. 2019;53:145-60.

2. Pacheco $\mathrm{S}$, Tejos R, Rodríguez J, Briceño

E, Guerra JF, Martínez J, et al. Tratamiento quirúrgico de las lesiones iatrogénicas de la vía biliar poscolecistectomía. Rev Chil Cir. 2017;69:202-6.

3. Zemour J, Marty M, Lapuyade B, Collet D, Chiche L. Gallbladder tumor and pseudotumor: Diagnosis and management. J Visc Surg. 2014;151:289-300.

4. van de Graaf FW, Zaïmi I, Stassen LPS, Lange JF. Safe laparoscopic cholecystectomy: A systematic review of bile duct injury prevention. Intl J Surg. 2018;60:164-72.

5. Eikermann M, Siegel R, Broeders I, Dziri $\mathrm{C}$, Fingerhut A, Gutt $\mathrm{C}$, et al. Prevention and treatment of bile duct injuries during laparoscopic cholecystectomy: The clinical practice guidelines of the European Association for Endoscopic Surgery
(EAES). Surg Endosc. 2012;26:3003-39.

6. Keplinger KM, Bloomston M. Anatomy and embryology of the biliary tract. Surg Clin N Am. 2014;94:203-17.

7. Pilgrim CHC, Usatoff V, Evans P. Consideration of anatomical structures relevant to the surgical strategy for managing gallbladder carcinoma. Eur J Surg Oncol. 2009;35:1131-6.

8. Cachoeira E, Rivas A, Gabrielli C. Anatomic Variations of Extrahepatic Bile Ducts and Evaluation of the Length of Ducts Composing the Cystohepatic Triangle. Int J Morphol. 2012;30:279-83.

9. Hiatt J, Gabbay J, Busuttil R. Surgical Anatomy of the Hepatic Arteries in 1000 Cases. Ann Surg. 1994;220:50-2.

10. Suzuki M, Akaishi S, Rikiyama T, Naitoh T, Rahman MM, Matsuno S. Laparoscopic cholecystectomy, Calot's triangle, and variations in cystic arterial supply. Surg Endosc. 2000;14:141-4.

11. Singh M, Prasad N. The anatomy of Rouviere's sulcus as seen during laparoscopic cholecystectomy: A proposed classification. J Minim Access Surg. 2017;13:89-95.

12. Hugh TB. New strategies to prevent laparoscopic bile duct injury - Surgeons can learn from pilots. Surgery 2002; 132:826-35.

13. Strasberg SM, Brunt LM. Rationale and Use of the Critical View of Safety in Laparoscopic Cholecystectomy. J Am Coll Surg. 2010;211:132-8.

14. Wakabayashi G, Iwashita Y, Hibi T, Takada T, Strasberg SM, Asbun HJ, et al. Tokyo Guidelines 2018: surgical management of acute cholecystitis: safe steps in laparoscopic cholecystectomy for acute cholecystitis (with videos). J Hepatobiliary Pancreat Sci. 2018;25:73-86.

15. Booij K, De Reuver P, Nijsse B, Busch O, Van Gulik T, Gouma D. Insufficient safety measures reported in operation notes of complicated laparoscopic cholecystectomies. Surgery 2014;155:3849.

16. Nijssen MAJ, Schreinemakers JMJ, Meyer Z, Van Der Schelling GP, Crolla 
RMPH, Rijken AM. Complications after laparoscopic cholecystectomy: A video evaluation study of whether the critical view of safety was reached. World J Surg. 2015;39:1798-803.

17. Honda G, Iwanaga T, Kurata M, Watanabe F, Satoh H, Iwasaki K. The critical view of safety in laparoscopic cholecystectomy is optimized by exposing the inner layer of the subserosal layer. J Hepatobiliary Pancreat Surg. 2009;16:445-9.

18. Pucher PH, Brunt LM, Fanelli RD, Asbun HJ, Aggarwal R. SAGES expert Delphi consensus: critical factors for safe surgical practice in laparoscopic cholecystectomy. Surg Endosc. 2015;29:3074-85.

19. Hope W, Fanelli R, Walsh D, Narula V, Price R, Stefanidis D, et al. SAGES clinical spotlight review: intraoperative cholangiography. Surg Endosc. 2017;31:2007-16.

20. Soper N, Dunnegan D. Routine versus selective intraoperative cholangiography during laparoscopic cholecystectomy. W J Surg. 1992;16:1133-40.

21. Van Manen L, Tummers QRJG, Inderson A, Bhalla A, Vahrmeijer AL, Bonsing $\mathrm{BA}$, et al. Intraoperative detection of the remnant cystic duct during robot-assisted surgery using near-infrared fluorescence imaging: A case report. BMC Surg. 2019;19:1-5.

22. Van Den Bos J, Schols RM, Luyer MD, Van Dam RM, Vahrmeijer AL, Meijerink WJ, et al. Near-infrared fluorescence cholangiography assisted laparoscopic cholecystectomy versus conventional laparoscopic cholecystectomy (FALCON trial): Study protocol for a multicentre randomised controlled trial. BMJ Open 2016;6:1-9.

23. Osayi SN, Wendling MR, Drosdeck JM,
Chaudhry UI, Perry KA, Noria SF, et al. Near-infrared fluorescent cholangiography facilitates identification of biliary anatomy during laparoscopic cholecystectomy. Surg Endosc. 2015;29:368-75.

24. Dip F, LoMenzo E, Sarotto L, Phillips E, Todeschini H, Nahmod M, et al. Randomized Trial of Near-infrared Incisionless Fluorescent Cholangiography. Ann Surg. 2019;270:992-9.

25. Michael Brunt L, Deziel DJ, Telem DA, Strasberg SM, Aggarwal R, Asbun H, et al. Safe cholecystectomy multi-society practice guideline and state-of-the-art consensus conference on prevention of bile duct injury during cholecystectomy. Ann Surg. 2020; 272:3-23.

26. Strasberg S, Pucci M, Brunt L, Deziel D. Subtotal Cholecystectomy-"Fenestrating" vs "reconstituting" Subtypes and the Prevention of Bile Duct Injury: Definition of the Optimal Procedure in Difficult Operative Conditions. J Am Coll Surg. 2016;222:89-96.

27. Dissanaike S. A Step-by-Step Guide to Laparoscopic Subtotal Fenestrating Cholecystectomy: A Damage Control Approach to the Difficult Gallbladder. J Am Coll Surg. 2016;223:e15-8.

28. Jara G, Rosciano J, Barrios W, Vegas L, Rodríguez O, Sánchez R, et al. Colecistectomía laparoscópica subtotal como alternativa quirúrgica segura en casos complejos. Cir Esp. 2017;95:465-70.

29. Sari YS, Tunali V, Tomaoglu K, Karagöz B, Güneyi A, Karagöz I. Can bile duct injuries be prevented? "A new technique in laparoscopic cholecystectomy”. BMC Surg. 2005;5:4-7.

30. Krähenbühl L, Sclabas G, Wente MN, Schäfer M, Schlumpf R, Büchler MW. Incidence, risk factors, and prevention of biliary tract injuries during laparoscopic cholecystectomy in Switzerland. World J Surg. 2001;25:1325-30.

31. Archer SB, Brown DW, Smith CD, Branum GD, Hunter JG. Bile duct injury during laparoscopic cholecystectomy: Results of a national survey. Ann Surg. 2001;234:549-59.

32. Ruiz F, Ramia J, García-Parreño J, Figueras J. Lesiones iatrogénicas de la vía biliar. Cir Española 2010;88:211-21.

33. Barbier L, Souche R, Slim K, Ah-Soune P. Long-term consequences of bile duct injury after cholecystectomy. J Visc Surg. 2014;151:269-79.

34. Roy PG, Soonawalla ZF, Grant HW. Medicolegal costs of bile duct injuries incurred during laparoscopic cholecystectomy. HPB 2009;11:130-4.

35. Sarmiento J, Farnell M, Nagorney D, Hodge D, Harrington J. Quality-of-Life Assessment of Surgical Reconstruction After Laparoscopic CholecystectomyInduced Bile Duct Injuries. Arch Surg. 2004;139:483.

36. Perera T, Silva M, Hegab B, Muralidharan V, Bramhall S, Mayer D, et al. Specialist early and immediate repair of postlaparoscopic cholecystectomy bile duct injuries is associated with an improved long-term outcome. Ann Surg. 2011;253:553-60.

37. Stewart L, Way L. Bile duct injuries during laparoscopic cholecystectomy. Arch Surg. 1995;130:1123-9.

38. Brunt M, Deziel D, Telem D, Strasberg S, Aggarwal R, Asbun H, et al. Safe cholecystectomy multi-society practice guideline and state-of-the-art consensus conference on prevention of bile duct injury during cholecystectomy. Ann Surg. 2020;272:3-23. 